\title{
On Word Formation of Computer English Vocabulary
}

\author{
Sun Wentao
}

Linyi University, Yishui, Shandong, 276400

\section{Keywords: Computer; English vocabulary; Word formation}

\begin{abstract}
With continuous development of computer technology in our country, lot of computer English vocabulary appeared in the process, and computer English vocabulary has a subtle relationship with general English. Computer English vocabulary refers to the computer related English words created by word-formation way of general English. There are many types of computer English vocabulary word-building ways, such as affixation, conversion, abbreviation, etc. The main characteristic of word formation is flexible usage, simple and clear. Part of computer English vocabulary constitutes new ideas through metaphor of old words. For these words, we want to understand its basic meaning and its meaning in computer technology.

With continuous development of society, network information age arrives. There are more and more education academic exchanges between countries. English is an important carrier of academic exchanges. According to a survey, in computer, electronics, automation and some science majors, more than $85 \%$ of the data are presented in the form of English. In computer development, computer professional English vocabulary also comes in different forms. In order to make people better master computer English vocabulary and make people flexibly use them when using computer, we have to study word-formation of the words and make people have a better understanding of word meaning.
\end{abstract}

\section{THE CHARACTERISTICS OF COMPUTER ENGLISH VOCABULARY}

Computer English is part of science and technology English. Science and technology English is divided into two kinds: one kind is professional or academic vocabulary terms. We call it pure science and technology. The other kind is the vocabulary for different professions usage, but it has different meanings in different professions, and we can call it general science and technology. Computer English vocabulary mostly belongs to the latter, scientific vocabulary. Computer English vocabulary are most common words related to People's Daily life, in English vocabulary, which are given new meanings on semantic changes in simple and clear form so people can very good master, which ensures the efficiency of information transfer. For instance, chip is translated into "thin" in daily life, but in computer English it is translated into "a small piece of silicon that has a set of complicated electrical connections on it and is used to store and process information in computers"; Program in daily life is translated into "courses", but in computer English it is translated into "a set of instructions given to a computer to make it perform an operation". There are many words like them, such as: bus (life-bus; computer-circuit); boot (life-guide; computer-initialization); keyboard (life-input; computer-keyboard), and so on. The characteristic of computer English vocabulary is that there is more scientific vocabulary. [1]

Computer English vocabulary has another characteristic that is to make words by using old vocabulary with new meaning. English vocabulary development process is related to the change of the society. In order to meet the demand of society development, some old words will get a series of changes. Some things have the same characteristics with other things in such aspects as behavior, phenomenon, so vocabulary can also give the behavior and phenomenon of one thing a name of behavior and phenomena of other things, such as metonymy, metaphor, etc. Computer English vocabulary takes the basis of semantic change and modification. Innovate meaning of words and make them with new semantics. Menu, for example, the original meaning is a list of food, while in computer English vocabulary, it is a list of instructions or program; Bug originally means insects, which means failure in computer English vocabulary. This word is produced by the fault happened 
when a moth flew to a traditional computer. Memory in computer vocabulary means stored program symbol or a part of the data. Its true meaning has bigger difference from (Memory), and it uses a metaphor, because computer is without the function of human Memory, and also it can't actually read and write, so it is the assumption that it can read information from the Internet or record information like people. [2]

\section{WORD FORMATION OF COMPUTER ENGLISH VOCABULARY}

Part of computer English vocabulary is not in professional term dictionary, and most of these words are reconstructed vocabulary, such as uninstall, undelete, resetup, etc. There is a law for English words, which is word-formation. As long as one masters this method, computer English vocabulary study is quite easy. Commonly used computer English vocabulary word-building ways are mainly transformation method, abbreviation method, synthesis, and so on.

A. Synthesis

Compound words in computer English vocabulary are a new kind of word formation, which are the new vocabulary mainly based on two or more words according to scientific order structure. The development of compound words in English plays an important role in the process, which positively adds a lot of English words. In computer English vocabulary, compound words are the majority and are presented in fixed form, use fewer hyphen, and have significant meaning. Most of them are formed by superposition as independent meanings of new words. In computer English vocabulary, adjectives + verbs, adjectives + adverbs, adjective + noun have strong magnetic force. For example, upload (up adjective + noun load), download (down adjective + load noun), login (log adjective + in noun), and so on. There are more compound word consisting of a noun + noun, such as diskcopy (copy all), letterbomb (lash bomb), webmaster (network specialist), filename (file name) and so on. Some adjectives are verbs like object-oriented adjective, like fade-in, download, packup. There are other compound words produced by adding suffixes to words, and change the nature and meaning of words, such as phase advancer (electronic phase displacement), pilot oscillator (channel oscillator), etc. [3]

B. Affix method

Affixation method is one of derived methods, which uses derivational affixes in roots to constitute a new vocabulary. According to the different positions of affix in the vocabulary, these words can be divided into two forms, prefix type and suffix type. Popular affix vocabulary has been widely used, but some are gradually forgotten by people.

In computer English vocabulary, there are prefixes which have strong ability of word-formation, which mainly include: re - retry, refresh, replay, rehyphenation, pre - preprocessor, prescan, preview, System - the Internet, interface, interactive, etc.; Tel - telenet, telecommuter (remote workers), etc.; [4]

In computer English vocabulary, suffixes having strong ability of word-formation mainly include: - er - explorer, server, - or - accelerator, monitor, colorize, minimize, etc.

\section{Conversion method}

When one kind has been converted into another, there is a connection converted the meaning of word meaning and the transformation. For example: reject (n.a product that has been rejected because there is something wrong with it), after conversion to reject (v. refuse); Time (n.), after conversion to time (v. to time). [5]

\section{Abbreviation}

Abbreviation method refers to the cutting of original words, split the letters and form new words, or unite the original initials into one word in vocabulary words or more letters to replace the original words. Acronym's informationization plays an important role in today's digital society. It has the advantages of simple usage. Such as the well-known World Wide Web (WWW); Personal Computer (PC); Microsoft Disk Operating System (MS-DOS), hypertext transfer protocol (HTTP), Central Processing Unit (CPU), etc.; There are some online words which are similar to cable language, for example, BTW - by the way, FYI - for your information, and so on. [6]

E. Old words new meaning 
The development of computer English vocabulary is associated with society development, and the old vocabulary will change their meaning in order to meet the demand of the development of the society. In computer English vocabulary, there are a lot of new words which are made from old words change. Such as register, it means registration or records in daily life, but register in computer vocabulary means memory; boot means kick in daily life, but in computer vocabulary, this action is an extension, and means to turn on computer power supply and start the computer and machine, and make the program work; Bulletin board in daily life means boards, and it extends in computer vocabulary and means electronic bulletin boards. Computer English vocabulary mostly comes from commercial terms, office terms and communication terms. Their only different is electronic function. Crash is mainly system problem which results in computer can't work in accordance with the normal instruction. File refers to the electronic version of the stored information inside the computer; Floppy disk is used to store information and documents; Information superhighway is the highway extension, which refers to the global computer network system through Internet connection; Mouse is a hand control device similar to a mouse; Virus gets extended by medicine, and the computer virus is like the virus in human body. Once there is computer virus, it cannot run normally and the files are damaged. [7]

\section{CONCLUSION}

Through the above illustration, we can learn that computer English vocabulary word formation is more and more simple and clear, the meaning included is also more and more simple, which can also be accepted by people in nowadays society. English is not our native language. When we study computer and other science and engineering major, we can always meet these English words, so we should not only fully understand the real meaning of them, but understand their meanings in computer or other science and engineering majors so that we can quickly learn computer technology.

\section{REFERENCE}

[1] Liu Hangying. Computer English terms word-formation method and Chinese translation method [D]. Shandong Normal University, 2013.

[2] Chang Yuli, Sun Fang. Study on word-formation of computer English vocabulary [J]. Microcomputer Applications, 2009, 25 (9) : 61-62.

[3] Peng Tao. Computer professional English vocabulary teaching [J]. Journal of Computer Education, 2010 (10) : 107-110.

[4] Mo Jia. On computer English vocabulary characteristics [J]. Science and Technology Plaza, 2006 (5) : 109-110.

[5] Guo Jiao, Yan Pei. On computer English vocabulary composition [J]. Journal of Exam Weekly, 2013 (70) : 76-76.

[6] Yu Zi. Metaphor and language innovation coexist---- computer English word-formation characteristics study [J]. Journal of Product Safety and Recall, 2003 (2) : 26-28.

[7] Zhang Jinsong. Computer professional English vocabulary characteristics and structure mode [J]. Journal of Information Science and Technology, 2010 (8). 